\title{
A controlled double blind study of azathioprine in the management of Crohn's disease
}

\author{
S Candy, J Wright, M Gerber, G Adams, M Gerig, R Goodman
}

\begin{abstract}
While immunosuppressive agents are used widely in the management of Crohn's disease, their efficacy has not been well established in randomised controlled trials. This study was designed to examine whether azathioprine increases remission rate when used in conjunction with a diminishing dose regimen of prednisolone over a period of 12 weeks. It further examined whether azathioprine offers any therapeutic advantage over placebo in the maintenance of remission in Crohn's disease over a period of 15 months. Sixty three patients with active Crohn's disease were treated with a 12 week diminishing dose of prednisolone and at the same time entered into a randomised, double blind 15 month trial of either azathioprine $(2.5 \mathrm{mg} / \mathrm{kg})$ or placebo. Remission rates between the two groups were compared at 12 weeks and at 15 months. There was no significant difference in the proportion of patients who had achieved and maintained remission by week 12 but at 15 months there was a highly significant difference in the proportion of patients in remission (42\% receiving azathioprine $v 7 \%$ receiving placebo), $\mathbf{p}=0.001$. Using life tables this beneficial effect was reflected as the difference in the median number of days on the trial $(p=0 \cdot 02)$. There were significantly greater decreases over the trial period in the median erythrocyte sedimentation rate, $C$ reactive protein, and leucocyte count in the azathioprine group. There were no cases of severe bone marrow suppression or clinical pancreatitis. In conclusion, azathioprine offers a therapeutic advantage over placebo in the maintenance of remission in Crohn's disease.

(Gut 1995; 37: 674-678)
\end{abstract}

Gastrointestinal Clinic, Department of Medicine, University of Cape Town and Groote Schuur Hospital, South Africa $S$ Candy J Wright

M Gerber

$G$ Adams

$M$ Gerig

R Goodman

Correspondence to: Dr S Candy, Groote Schuur Hospital, Gastro-intestinal Clinic, E23, E-Floor, NGSH, Observatory, Cape Town 7925, South Africa.

Town 7925, South Africa. 16 March 1995
Keywords: Crohn's disease, azathioprine, immunosuppression.

The aetiology of Crohn's disease is unknown and as a result treatment options are limited. Corticosteroids, despite their side effects, remain the treatment modalities of choice. Intensive research has been directed at finding a safe and effective alternative to corticosteroids, particularly for use in resistant Crohn's disease and in the maintenance of remission. Immunosuppressive drugs such as azathioprine and its active metabolite, 6-mercaptopurine, have been used extensively in these clinical settings but their efficacy has yet to be proved unequivocally in controlled trials. A recent meta-analysis of all published single drug controlled trials concluded that no single drug affords a therapeutic advantage in the maintenance of remission in Crohn's disease. ${ }^{1}$ This study was designed to examine the combined effect of azathioprine and prednisolone in inducing remission in active Crohn's disease and further to test the efficacy of azathioprine alone in the maintenance of remission.

\section{Methods}

PATIENTS

The study was approved by the Ethics and Research Committee of the Faculty of Medicine, Groote Schuur Hospital. Each patient gave written informed consent before entry into the study. Patients between the ages of 15 and 65 years with confirmed Crohn's disease who were being followed up at the Groote Schuur Hospital Inflammatory Bowel Disease Clinic provided the source population. The inclusion criteria were a radiological or endoscopic diagnosis of Crohn's disease and the presence of active disease determined by a score of 200 or more on the Crohn's Disease Activity Index. ${ }^{2}$ Patients excluded were those with extensive previous surgery for Crohn's disease or those with symptoms suggestive of a mechanical obstruction that might require imminent surgery. Recent immunosuppression, compromised hepatic function, pregnancy, and lactation were further exclusions. At entry, age, the time since onset of symptoms and the time since diagnosis, smoking status, extent of disease, previous surgery, previous medical management (specifically any recent corticosteroid treatment), disease activity (reflected by the CDAI, erythrocyte sedimentation rate (ESR), serum $C$ reactive protein (CRP) and orosomucoid) and the presence or absence of perianal disease and fistulas were recorded.

\section{STUDY DRUG}

The azathioprine formulation was in the form of film coated tablets, trade name Imuran (Wellcome Pty, Kempton Park, South Africa).

STUDY DESIGN

This was a single centre, two phase, double blind, placebo controlled trial that compared the combined effect of prednisolone and azathioprine $v$ prednisolone and placebo in the treatment of active Crohn's disease over a 
period of 12 weeks (phase 1). The second phase (phase 2) compared azathioprine alone $v$ placebo in the maintenance of remission induced in phase 1 over a further 12 months.

Patients were recruited as they presented over a 36 month period. They were stratified according to disease extent into three groups; group 1: those with only ileal disease, group 2: those with ileocolitis, and group 3: those with isolated colonic disease. They were then randomised to either active medication or placebo to ensure equal representation of disease extent within the treatment groups. At entry a baseline assessment of the previous weeks' symptoms was made and the patients were examined clinically. Blood was drawn to measure ESR, serum CRP and orosomucoid, as well as comprehensive haematological and biochemical indices.

Visits were scheduled weekly for the first month, twice weekly for the second month, and monthly thereafter for the remainder of the trial or until withdrawal. Predetermined withdrawal criteria were: failure to achieve remission within the first 12 weeks (defined by a CDAI of $>150$ ), relapse (defined as a CDAI $>175$ ) at any stage thereafter, patient request, discretion of the investigators and bone marrow suppression, defined as a haemoglobin of less than $7.0 \mathrm{~g} / \mathrm{dl}$, a white cell count of less than $2 \times 10^{9} / 1$ or a platelet count lower than $100 \times 10^{9} /$. Haematological and biochemical indices were monitored independently by a physician not involved in the clinical assessment. Decisions regarding drug dose adjustment were relayed to the patient by the nurse practitioner who was also responsible for symptom assessment, drawing blood, counting remaining tablets, and issuing the trial medications.

The physician responsible for the clinical examination recorded any reported side effect or adverse event experienced by the trialist since the last visit and noted any concomitant medication taken.

All medications for Crohn's disease were stopped at entry except for antidiarrhoeal drugs, which too were recorded at each visit.

\section{STUDY DRUG ADMINISTRATION}

At day 1, all randomised patients were given prednisolone at a starting dose of $1 \mathrm{mg} / \mathrm{kg} /$ day. They were requested to decrease this dose by $5 \mathrm{mg}$ per week to zero at the end of week 12 . Where a patient weighed less than $60 \mathrm{~kg}$, the starting dose was maintained for a longer period of time before decreasing to ensure that prednisolone was continued throughout phase 1. Similarly, if the weight was greater than 60 $\mathrm{kg}$, the dose was reduced more rapidly over the first two weeks to ensure completion of prednisolone by the end of week 12 .

Also on day 1 , patients were presented with tablets of identical appearance (scored ' $50 \mathrm{mg}$ ' on one side and 'Imuran' on the other). The dose was calculated at $2.5 \mathrm{mg} / \mathrm{kg}$ but each patient was asked to take only one tablet (50 $\mathrm{mg}$ ) per day for the first week as a preventative measure against idiosyncratic leucopenia in those randomised to azathioprine. If the leuco- cyte count at week 1 was normal, the patient was advised to take the full dose $(2.5 \mathrm{mg} / \mathrm{kg}$ to the nearest $50 \mathrm{mg}$ ) as a single daily administration. Where the leucocyte count dropped to less than $4 \times 10^{9} /$, the dose of trial drug was reduced, usually by $50 \mathrm{mg}$ initially. The count was then monitored closely. In the event of good recovery, the original dose was reinstated. Compliance was assessed at each subsequent visit by means of a tablet count.

\section{EVALUATION METHODS}

Patients were asked to keep a diary charting stool number and consistency, abdominal pain, general wellbeing, concomitant medication, and any adverse clinical experiences. There were two evaluation points; the first being at cessation of prednisolone at 12 weeks and the second at the end of 15 months. The primary measure of therapeutic response was in each case the proportion of patients in remission. Remission was defined at 12 weeks as a CDAI of $<150$ and at 15 months as a CDAI $<175$. Withdrawal for any reason other than clinical relapse was considered therapeutic failure.

Secondary measures of therapeutic response were the median change in CDAI, ESR, serum CRP, serum orosomucoid concentration, and leucocyte count between the first and last visits.

The difference in leucocyte count between baseline and final visit was compared for responders $v$ non-responders within the azathioprine group to ascertain whether induction of leucopenia predicts clinical response.

\section{STATISTICAL METHODS}

All patients randomised into the trial were analysed - that is, analysis was performed on an intention to treat basis. No data necessary for the calculation of the CDAI were missing. A two-tailed $\chi^{2}$ test was used to analyse the difference in the proportion of patients in remission at week 12 and at trial end. A Wilcoxon test was used to compare the median change from baseline to last visit in CDAI, ESR, CRP, orosomucoid concentration, and leucocyte count between the two groups.

The calculated 'relative risk' - that is, the relative protective effect proferred by azathioprine was adjusted for key potential confounders using the Mantel-Haenszel calculation. The simultaneous influence of a number of covariates on the association of treatment status and outcome was examined by means of the logistic regression procedure of SAS (Ver 6.08). A Kaplan-Meier life table analysis was used to compute the median time to relapse. Analysis of variance was used to compare the median time to relapse by disease extent.

\section{Results}

PATIENTS

A total of 63 patients were randomised to receive either placebo or azathioprine in 
TABLE I Baseline characteristics

\begin{tabular}{lcc}
\hline Baseline characteristic & Azathioprine $(n=33)$ & Placebo $(n=30)$ \\
\hline Male (\%) & $7(21)$ & $11(37)$ \\
Female (\%) & $26(79)$ & $19(63)$ \\
Age (y) (median and range) & $33 \cdot 9(15-60)$ & $31 \cdot 8(21-62)$ \\
Weight (kg) (median and range) & $53 \cdot 1(46 \cdot 7-62)$ & $57 \cdot 5(48-64)$ \\
Duration of symptoms (y) (median and range) & $3 \cdot 7(0 \cdot 6-19 \cdot 3)$ & $4 \cdot 7(0 \cdot 2-19 \cdot 4)$ \\
Time since diagnosis (y) (median and range) & $2 \cdot 6(0 \cdot 1-19 \cdot 0)$ & $3 \cdot 7(0 \cdot 1-18 \cdot 7)$ \\
Extent (I:IC:C) & $8: 20: 5$ & $6: 19.5$ \\
Previous surgery (\%) & $6(18)$ & $8(27)$ \\
Episode in previous 6-12 months (\%) & $28(85)$ & $21(70)$ \\
Corticosteroids in previous 6-12 months (\%) & $22(67)$ & $19(63)$ \\
No previous corticosteroids (\%) & $5(15)$ & $2(7)$ \\
Smoking status (\%) & $22(67)$ & $20(67)$ \\
Severe disease (CDAI >300) (\%) & $17(52)$ & $13(43)$ \\
CDAI & $301(264-358)$ & $282(240-356)$ \\
ESR (mm/h) & $45(20-59)$ & $26(15-42)$ \\
CRP (mg\%) & $5 \cdot 4(2 \cdot 9-7 \cdot 3)$ & $3 \cdot 9(2 \cdot 8-5 \cdot 3)$ \\
Orosomucoid (g/l) & $1 \cdot 85(1 \cdot 27-2 \cdot 52)$ & $1 \cdot 79(1 \cdot 47-2 \cdot 52)$ \\
Leucocyte count $\left(\times 10^{\%} / 1\right)^{\star}$ & $9 \cdot 0(7 \cdot 0-10 \cdot 9)$ & $10 \cdot 0(5 \cdot 6-12 \cdot 6)$ \\
\hline
\end{tabular}

${ }^{\star}$ Median and interquartile range. $\mathrm{I}=$ ileal, $\mathrm{IC}=$ ileocolitis, $\mathrm{C}=$ colonic disease.

addition to a 12 week course of prednisolone. The treatment and placebo groups were similar with respect to the basic demographic data (Table I).

EARLY DISCONTINUATION OF THERAPY

At 12 weeks, 20 of 63 randomised patients $(32 \%)$ had been withdrawn: nine of $33(27 \%)$ in the azathioprine group and 11 of $30(37 \%)$ in the placebo group.

Overall, $47(74 \cdot 6 \%)$ of those randomised withdrew before the end of the trial: 28 of 30 $(93 \%)$ in the placebo group and 19 of 33 $(58 \%)$ in the azathioprine group. Table II gives the reasons for withdrawal.

\section{PRIMARY EFFICACY PARAMETERS}

Remission at 12 weeks

There was no significant difference between the proportions of patients who had achieved and maintained remission at 12 weeks: 24 of $33(73 \%)$ in the azathioprine group $v 19$ of 30 $(63 \%)$ receiving placebo $(p=0 \cdot 6)$.

\section{Remission at 15 months}

There was a highly significant difference between the proportions of patients who were still in remission at the end of the trial period: 14 of $33(42 \%)$ in the azathioprine group $v$ two of $30(7 \%)$ receiving placebo, $(p=0.001)$. 'Relative risk' (relative protective effect of azathioprine $v$ placebo was 6.36 (95\% confidence intervals (CI) 1.6 to $25 \cdot 7$ ). This ratio remained clinically and statistically significant after adjusting for disease severity $(R R=6.84 \quad(1.68$ to

TABLE II Reasons for withdrawal

\begin{tabular}{lll}
\hline & \multicolumn{2}{l}{ Treatment group } \\
\cline { 2 - 3 } Reason & $\begin{array}{c}\text { Placebo } \\
(n=28)\end{array}$ & $\begin{array}{l}\text { Azathioprine } \\
(n=19)\end{array}$ \\
\hline No response phase I & 9 & 7 \\
Default & 1 & 2 \\
$\quad$ In remission & 0 & 1 \\
$\quad$ Not in remission & 2 & 0 \\
Clinical side effect & 0 & 0 \\
Haematological side effect & 0 & 1 \\
Patient request & 13 & 7 \\
Clinical relapse (phase 2) & 3 & 1 \\
Other, for example, pregnancy & & \\
\hline
\end{tabular}

24.93), time since diagnosis $(\mathrm{RR}=6.48$ ( 1.68 to $24.93))$, and sex ( $R R=6.9(1.65$ to $28 \cdot 8))$.

The influence of a number of variables was further examined with logistic regression using a stepwise procedure, without changing the result. The analysis was repeated after excluding defaulters and those withdrawn for reasons other than relapse; the result remained clinically and statistically highly significant.

The question of effect modification by disease extent was considered by stratifying into three groups; ileitis $(n=14)$, ileocolitis $(n=39)$, and colitis $(n=10)$. Table III gives a summary of this analysis. Although the category 'ileocolitis' seems to predispose to a more favourable outcome (OR $=14.7$ (95\% CI $2 \cdot 2$ to $96 \cdot 5$ ), the small numbers in the other groups and wide confidence intervals make analysis meaningless.

\section{Life table analysis}

Overall - the median number of days on the trial was 263 (range 4-540) with azathioprine $v$ 139 (range 7-446) with placebo $(\mathrm{p}=0.02)$ (Figure).

Analysed by disease extent - the median number of days on the trial for those randomised to azathioprine was 345 where disease was restricted to the ileum, 249 for those with ileocolitis, and 233 for those with colitis alone $(p=0.57)$. Similarly, for those receiving placebo, the number of days on the trial were 96,147 , and 141 respectively $(p=0 \cdot 74)$.

\section{SECONDARY EFFICACY PARAMETERS}

At 12 weeks

There were no significant differences from baseline between the two groups with respect to CDAI $(p=0.2)$, CRP $(p=0.06)$ or orosomucoid $(p=0 \cdot 8)$. The median ESR increased by $1.5 \mathrm{~mm} /$ hour among those receiving prednisolone alone while it dropped by $17 \cdot 5$ $\mathrm{mm} /$ hour in those patients receiving azathioprine. This represents a highly significant difference in favour of azathioprine $(p=0.004)$.

\section{At 15 months}

Table IV lists the median differences in secondary efficacy parameters for trial commencement to trial end.

TABLE III Proportion of patients in remission at trial end by disease extent

\begin{tabular}{|c|c|c|}
\hline & Responders $(n=16)$ & Non-responders $(n=47)$ \\
\hline \multicolumn{3}{|l|}{ Total $(n=63)$} \\
\hline Azathioprine & 14 & 19 \\
\hline Placebo & 2 & $28^{\star}$ \\
\hline \multicolumn{3}{|l|}{ Ileitis $(n=14)$} \\
\hline Azathioprine & 4 & 4 \\
\hline Placebo & 1 & $5+$ \\
\hline \multicolumn{3}{|l|}{ Ileocolitis $(n=39)$} \\
\hline Azathioprine & 9 & 11 \\
\hline Placebo & 1 & $18 \ddagger$ \\
\hline \multicolumn{3}{|l|}{ Colitis $(n=10)$} \\
\hline Azathioprine & 1 & 4 \\
\hline Placebo & 0 & $5 \$$ \\
\hline
\end{tabular}




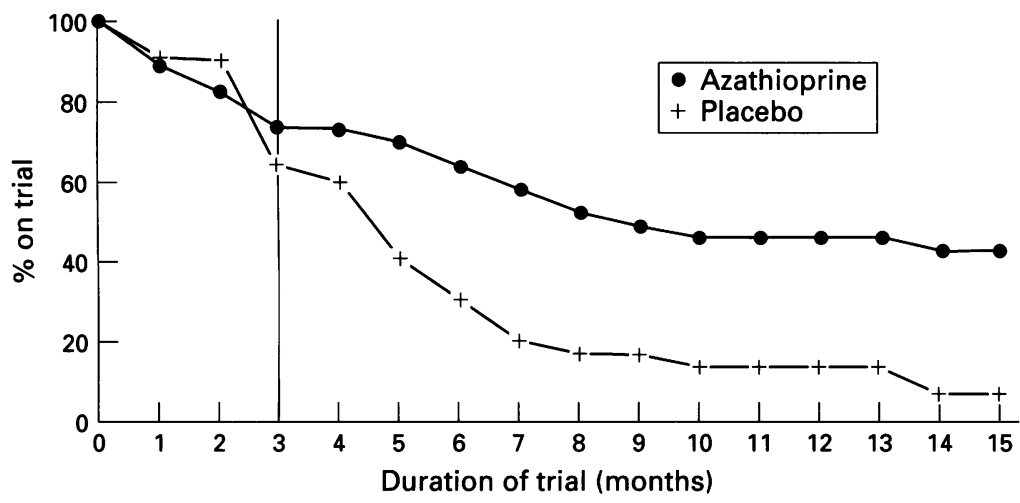

Life table representation.

\section{Leucocyte count}

The change in median white cell count was significantly different between the two groups. Median leucocyte count actually rose in the placebo group.

In those patients randomised to azathioprine, the white cell count at the final visit was significantly lower in those who responded - that is, those in remission at trial end - than those who did not $(p=0.005)$ (Table V). Nine of 13 patients who developed leucopenia sufficient to warrant a decrease in drug dose $v$ five of 20 who did not, were still in remission at the end of the trial $(\mathrm{OR}=6 \cdot 75(95 \% \mathrm{CI} 1 \cdot 15$ to $42 \cdot 7))$.

\section{Acute phase reactants}

The reduction in median values for ESR and CRP were significantly different between the two groups. For technical reasons final visit orosomucoid concentrations were performed in only 45 patients. The median drop in this index of disease activity was 0.58 in the azathioprine group $v 0.28$ in those receiving placebo. This difference did not reach statistical significance.

\section{$C D A I$}

As a result of the wide range in values, the median differences in CDAI were not significant between the azathioprine and placebo groups $(p=0 \cdot 06)$.

\section{Smoking}

To ascertain whether the rates of smoking were consistent with the published data for South Africa, ${ }^{3}$ the group was analysed by sex, population group, and smoking status. The prevalence of smoking among female patients in this trial was approximately double that expected

TABLE IV Change from baseline to trial end (median, interquartile range, and number of subjects)

\begin{tabular}{llllll}
\hline \multicolumn{7}{l}{ Treatment group } \\
\cline { 2 - 6 } & Subject $(n)$ & Azathioprine & Subject $(n)$ & Placebo & \\
\hline CDAI & 32 & $191 \cdot 5(45 \cdot 5-256 \cdot 5)$ & 30 & $50(-8-222)$ & $\mathrm{p}=0.06$ \\
ESR & 32 & $15 \cdot 5(1-31)$ & 30 & $-6 \cdot 5(-17-9)$ & $\mathrm{p}=0.0005$ \\
CRP & 31 & $30(0-53)$ & 30 & $0(-10-17)$ & $\mathrm{p}=0.003$ \\
Orosomucoid & 23 & $0 \cdot 58(0 \cdot 18-1 \cdot 06)$ & 22 & $0 \cdot 28(0 \cdot 14-0 \cdot 70)$ & $\mathrm{p}=0 \cdot 15$ \\
Leucocyte & 32 & $2 \cdot 8(0 \cdot 45-4 \cdot 85)$ & 30 & $-0 \cdot 8(-2 \cdot 8-3 \cdot 2)$ & $\mathrm{p}=0.02$ \\
Weight & 31 & $-2 \cdot 6(-10 \cdot 2-0)$ & 30 & $-3.0(-6.9-1)$ & $\mathrm{p}=0.9$ \\
\hline
\end{tabular}

A negative value shows an increase over trial period. in the general population; $70 \% v 30 \%$ for white women and $68 \% v 33 \%$ for women of mixed race. A less striking trend was found among male patients with prevalences of $57 \%$ $v 41 \%$ for white, and $64 \% v 50 \%$ for men of mixed race.

\section{Discussion}

In this placebo controlled study of 63 patients with active Crohn's disease, azathioprine offered a clinically significant advantage over placebo in the maintenance of remission induced by a three month course of diminishing dose prednisolone. No significant adjunctive role could be shown for this drug when used with prednisolone in the induction of remission over a period of 12 weeks. A significantly greater decrease in the ESR was noted in those receiving combination therapy, however.

Previous controlled trials of immunosuppression in Crohn's disease have shown conflicting results. ${ }^{4-11}$ Small numbers and differing methodologies make comparison of these studies difficult, though Pearson, in a recent meta-analysis, has shown a significant role for azathioprine and 6-mercaptopurine in both active and maintenance therapy. ${ }^{12}$ In a comparatively large randomised withdrawal study, O'Donoghue and colleagues found azathioprine at a dose of $2 \mathrm{mg} / \mathrm{kg} /$ day to be effective in the maintenance of remission. ${ }^{8}$ This early study has been criticised for having examined a subgroup of patients who had responded to the drug before withdrawal. The largest and perhaps most vigorous of these studies, the National Cooperative Crohn's Disease Study failed to show a significant role for azathioprine in either acute management (part 1) or as maintenance therapy (part 2). ${ }^{9}$ Their negative findings may be explained by the fact that during both parts of this trial, azathioprine was used as single drug therapy and mostly at the low dose of $1 \mathrm{mg} / \mathrm{kg}$. Interestingly, multivariate analysis of the maintenance trial, despite the overall negative findings, established that patients treated with corticosteroids before entry into the study, maintained remission for significantly longer than those who were not.

The largest controlled trial to find a positive role for immunosuppression in Crohn's disease was that of Present et al, which was published shortly after the findings of the NCCDS in $1980 .{ }^{10}$ In this two year double blind crossover study of 83 patients, the authors showed 6-mercaptopurine (the active metabolite of azathioprine) to be significantly superior to placebo. At a mean starting dose of $1 \mathrm{mg} / \mathrm{kg}$, patients receiving 6-mercaptopurine had a $67 \%$ overall improvement in symptoms compared with $8 \%$ receiving placebo. Their study showed convincing support for 6-mercaptopurine in the treatment of fistulas and as a corticosteroid sparing agent.

Ewe and colleagues who have published data to support the use of a combination regimen of azathioprine at a dose of $2.5 \mathrm{mg} / \mathrm{kg}$ and prednisolone in active Crohn's disease, 
TABLE V Median white cell count and interquartile range in subjects randomised to azathioprine. A comparison of those who completed the trial in remission (responders) with those who did not (non-responders)

\begin{tabular}{lllll}
\hline & Subjects $(n)$ & Baseline & Final visit & Difference \\
\hline Responders & 14 & $9 \cdot 3(7 \cdot 0-13 \cdot 0)$ & $4 \cdot 9(3 \cdot 9-5 \cdot 7)$ & $4 \cdot 3(2 \cdot 6-5 \cdot 9)$ \\
Non-responders & 18 & $8 \cdot 5(5 \cdot 6-10 \cdot 7)$ & $6 \cdot 8(5 \cdot 1-9 \cdot 0)$ & $1 \cdot 5(-0 \cdot 9-3 \cdot 0)^{\star}$
\end{tabular}

${ }^{\star} p=0.005$. The number of patients analysed was 32 not 33 as one was withdrawn on day 5 without final visit data.

concluded that patients went into remission more often and earlier and stayed in remission longer on the combined regimen. ${ }^{11} \mathrm{We}$ were unable to show this adjunctive role in phase 1 of our study. There are two possible explanations. Firstly, the protocol in Ewe's study allowed for the dose of prednisolone to be increased in the event of deterioration in symptoms on two occasions. In our study a CDAI score of more than 150 in phase 1 resulted in immediate withdrawal. Secondly, their trial period was four months in total compared with the three months of our phase 1. Assuming that azathioprine may take up to four months to become effective, ${ }^{13}$ a possible subgroup of late responders would have been lost in our study. Examination of the life table graph shows divergence of the group's relapse rates at approximately four months. It is interesting to note, however, that the median ESR was significantly lower at 12 weeks in those randomised to azathioprine. Though not statistically significant, there was a similar trend for CRP.

The rationale for not using azathioprine and 6-mercaptopurine as first line therapy in Crohn's disease has been the potentially serious side effects. Clinical pancreatitis, reportedly the commonest short-term complication $(3-7 \%)^{14}$ was not encountered in our study, though routine amylase and lipase tests were not performed. A single patient developed epigastric pain sufficient to warrant withdrawal from the study. Serum and urinary amylase values taken at the time of withdrawal were normal. The pain resolved spontaneously and at completion of trial analysis, the patient was found to have been randomised to placebo. Bone marrow suppression sufficient to warrant withdrawal from the study (see criteria in methods) was not encountered, but 13 of 33 patients in the azathioprine group did require a decrease in their drug dose as a result of a fall in leucocyte count below $4 \times 10^{9} / 1$. (All counts increased after diminution of dose.) Leucopenia did not occur during the combination prednisolone/azathioprine period and, where it did occur thereafter, there was no consistent time interval in which it developed. Despite small numbers, there was a highly significant negative correlation between leucocyte count and therapeutic outcome within the azathioprine group. Colonna et al in a retrospective study found a strong positive correlation between extent of drug induced leucopenia and clinical success of immunosuppressive therapy. ${ }^{15}$ Our study confirms this finding.

Under the influence of the beneficial findings of Korelitz et al, ${ }^{16}$ immunosuppression has been used extensively in fistulous disease. As radiological studies were not performed at entry and completion in this trial, success of therapy could not be ascertained for enteroenteral fistulas. In the two patients with rectovaginal fistulas receiving active medication, drainage diminished entirely during the trial but this may reflect firming of the stool rather than fistulous healing. The only patient with extensive perianal Crohn's randomised to azathioprine did not respond and was withdrawn before 12 weeks.

The association between smoking and Crohn's disease has been established epidemiologically. ${ }^{17}$ When analysed by sex and population group, the prevalence of smoking in our overall sample was higher than in the general population. ${ }^{3}$ Multivariate analysis, however, failed to show smoking status as an effect modifier in determining response to azathioprine.

The results of this trial add weight to the accumulating evidence for the use of a combination of azathioprine and short-term corticosteroids in active Crohn's disease. Further, our data suggest that azathioprine at a starting dose of $2.5 \mathrm{mg} / \mathrm{kg} /$ day is safe and effective in the longterm maintenance of remission induced by such a combination regimen.

Imuran and placebo tablets were kindly supplied by Wellcome SA Pty Ltd. We are grateful to Doctor Rodney Ehrlich for his help with the statistical analysis.

1 Salomon P, Kornbluth A, Aisenberg J, Janowitz HD. How effective are current drugs for Crohn's disease. A metaanalysis. F Clin Gastroenterol 1992; 14: 211-5.

2 Best WR, Bectel JM, Singleton JW, Kern F. Development of a Crohn's Disease Activity Index. Gastroenterology 1976; 70: 439-44.

3 Yach D, McIntyre D, Saloojee Y. Smoking in South Africa: the health and economic impact. Tobacco Control 1992; 1: 272-80.

4 Willoughby JMT, Kumar PJ, Beckett J, Dawson AM. Controlled trial of azathioprine in Crohn's disease. Lancet 1971; ii: 944-7.

5 Rhodes J, Bainton D, Beck P, Campbell H. Controlled trial of azathioprine in Crohn's disease. Lancet 1971; ii: 1273-6.

6 Klein M, Binder HJ, Mitchell M, Aaronson R, Spiro H. Treatment of Crohn's disease with azathioprine: a controlled evaluation. Gastroenterology 1974; 66: 916-22.

7 Rosenberg JL, Levin B, Wall AJ, Kirsner JB. A controlled trial of azathioprine in Crohn's disease. Dig Dis 1975; 20: 721-6.

8 O'Donoghue DP, Dawson AM, Powell-Tuck, Bown RL, Lennard-Jones JE. Double-blind withdrawal trial of azathioprine as maintenance treatment for Crohn's disease. Lancet 1978; ii: 955-7.

9 Summers RW, Switz DM, Sessions JT Jr, Becktel JM, Best WR, Kern F Jr, et al. National Cooperative Crohn's Disease Study: results of drug treatment. Gastroenterology 1979; 77: 847-69.

10 Present DH, Korelitz BI, Wisch N, Glass JL, Sachar DB Pasternack BS. Treatment of Crohn's disease with 6-mercaptopurine. A long-term randomised double-blind study. N Engl ₹ Med 1980; 302: 981-7.

11 Ewe K, Press AG, Singe CC, Stufler M, Ueberschaer B Hommel G, et al. Azathioprine combined with prednisolone or monotherapy with prednisolone in active Crohn's disease. Gastroenterology 1993; 105: 367-72.

12 Pearson DC, May GR, Fick GH, Sutherland LR. Azathioprine and 6-mercaptopurine in Crohn's disease: a Azathioprine and 6-mercaptopurine in Crohn's

13 O'Brien JJ, Bayless TM, Bayless JA. Use of azathioprine or 6 mercaptopurine in the treatment of Crohn's disease. 6 mercaptopurine in the treatment

14 Present DH, Meltzer SJ, Wolke A, Korelitz BI. Short- and long-term toxicity to 6-mercaptopurine in the treatment of inflammatory bowel disease. Ann Intern Med 1989; 3 641-9.

15 Colonna T, Korelitz BI. The role of leukopenia in the 6 mercaptopurine-induced remission of refractory Crohn's disease. Am $\mathcal{F}$ Gastroenterol 1994; 89: 362-6.

16 Korelitz BI, Present DH. Favorable effect of 6-mercaptopurine on fistulae of Crohn's disease. Dig Dis Sci 1985; 30 $58-64$

17 Calkins BM. A meta-analysis of the role of smoking in inflammatory bowel disease. Dig Dis Sci 1989; 34: 1841-54. 